\title{
Antenatally Diagnosed Binder Syndrome in Newhorn following Maternal Warfarin Intake in First Trimester
}

\section{JCR}

\author{
Renuka Malik, Garima Agarwal \\ Department of Obstetrics and Gynaecology, Post Graduate Institute of Medical Education \\ and Research and RML Hospital, New Delhi 110001 , India.
}

\begin{abstract}
:
The primary physical characteristic of Binder syndrome is midfacial hypoplasia and flattened nose associated with the absence of the anterior nasal spine. Binder syndrome is a rare developmental defect which has many etiologies including vitamin $\mathrm{K}$ deficiency which may occur following warfarin intake during first trimester. It is rare disorder occurring in less than 1 per 10,000 live births. Common differential diagnosis includes CDPR (chondrodysplasia punctata, rhizomelic type) and fetal warfarin syndrome. We hereby report a case diagnosed antenatally as Binder syndrome in a fetus of woman suffering from rheumatic heart disease, who took warfarin in first trimester because of mechanical mitral valve prosthesis. This case is presented to highlight the teratogenic potential of warfarin and importance of preconceptional counseling by cardiologist and obstetrician and also to suggest the role of vitamin $\mathrm{K}$ deficiency in facial dysmorphism in Binder Syndrome.
\end{abstract}

Key words: Drug Induced Abnormalities, Cataract, Pregnancy, Rheumatic Heart Disease, Vitamin K Deficiency, Warfarin.

\section{Introduction}

Binder syndrome is a rare congenital anomaly with multiple etiologies and multiple associated disorders which are poorly understood because of rarity. Warfarin is a long acting small molecule which crosses placenta and has a long half-life, detectable in blood even after one month of stopping. It is teratogenic when given at 6-9 weeks of pregnancy leading to facial dysmorphism and other skeletal defects which are due to its vitamin $\mathrm{K}$ antagonistic effect. Patient should be counseled regarding fetal risk which varies depending on the anticoagulant used during pregnancy [warfarin, unfractionated heparin (UFH) or low molecular weight heparin (LMWH)]. However, it may be seen that facial dysmorphism in Binder syndrome is generally also due to other causes. With advanced ultrasound machines, the typical features of absent nasal bone and maxillary hypoplasia can be diagnosed antenatally.

\section{Corresponding Author: Dr. Renuka Malik}

Email: renucam@yahoo.co.in

Received: December 16, 2015 | Accepted: January 6, 2016 | Published Online: January 25, 2016

This is an Open Access article distributed under the terms of the Creative Commons Attribution License (creativecommons.org/licenses/by/3.0)

Conflict of interest: None declared | Source of funding: Nil | DOl: http://dx.doi.org/10.17659/01.2016.0010 


\section{Case Report}

25 years old, second gravida, abortion 1, Hindu housewife, known case of rheumatic heart disease since last seven years presented to Gynecology OPD of PGIMER, RML Hospital, New Delhi at 15 weeks of gestation. Patient had mechanical mitral valve replacement done 4 years back in our hospital and was on tablet warfarin $6 \mathrm{mg}$ once daily, ecospirin $75 \mathrm{mg}$ and injection benzathaine penicillin 1.2MU 3 weekly. She had no preconception counseling or cardiology consultation since last 3 years. Patient was referred for cardiological consultation. Her INR (International normalized ratio) was 2.4. Anomaly ultrasound done at 20 weeks [Fig. 1] demonstrated nasal hypoplasia, flat facial profile, and prominent forehead suggesting Binder syndrome. After seeing the report of Binder syndrome in baby the cardiologist stopped warfarin and shifted to subcutaneous LMWH $40 \mathrm{IU}$, once a day. Patient continued to take ecospirn $75 \mathrm{mg}$ and injection

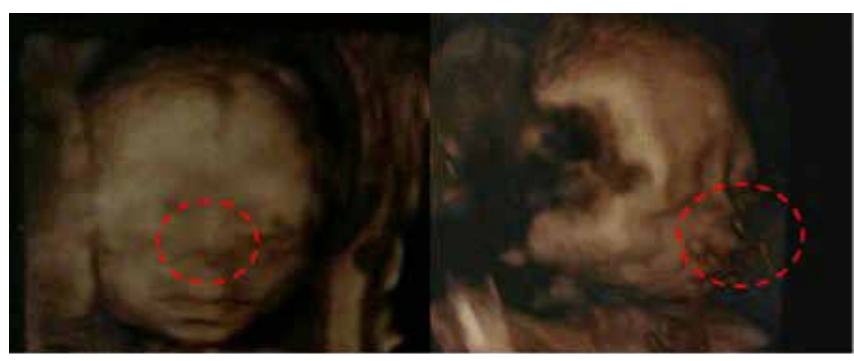

Fig.1: 3-D antenatal ultrasound scans showing nasal hyperplasia \& flat fronto-nasal angle in AP and lateral view.

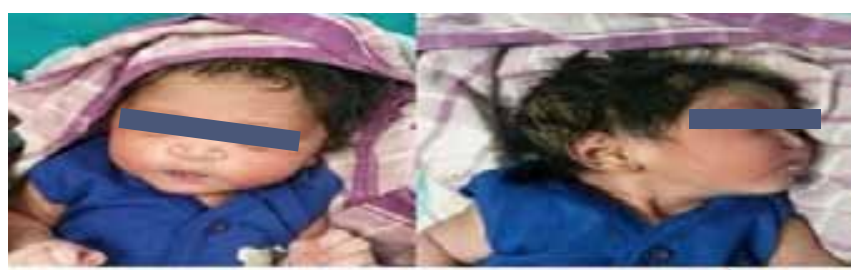

Fig.2: Front and lateral profile of the new born showing Binder's phenotype. benzathaine penicillin three weekly. Patient was in NYHA class II and remained so till term. All her antenatal blood and urine investigations were normal. Repeat echocardiography done at 20 weeks and 36 weeks revealed good functioning of mitral prosthetic valve with mild PAH (pulmonary artery hypertension). She was jointly assessed by cardiologist, anesthesist and obstetrician at 37 weeks and planned for spontaneous vaginal delivery. Ecospirin was stopped at 39 weeks. At 40 weeks, when she complained of labor pains, LMWH was stopped. Patient delivered 12 hours after onset of labor. A female baby, weighing $2.7 \mathrm{~kg}$ was delivered with a normal APGAR score. Following delivery, she developed $5 \times 8 \mathrm{~cm}$ vulval hematoma that was drained under general anesthesia. Warfarin was started in dose of $6 \mathrm{mg}$, 24 hours after evacuation. Her post-partum period in hospital was uneventful there-after.

Examination of the baby revealed certain facial abnormalities. There was absent nasal bone with maxillary bone hypoplasia [Fig.2,3], semi-lunar alae nasi and convex upper lip. A joint consultation with pediatrician, pediatric ophthalmologist and plastic surgery department was taken. Neonatologist tested for vision and hearing which were found to be normal. Infantogram did not reveal any shortening or stippling of epiphysis of long bones [Fig.4]. The neonatologist did not feel

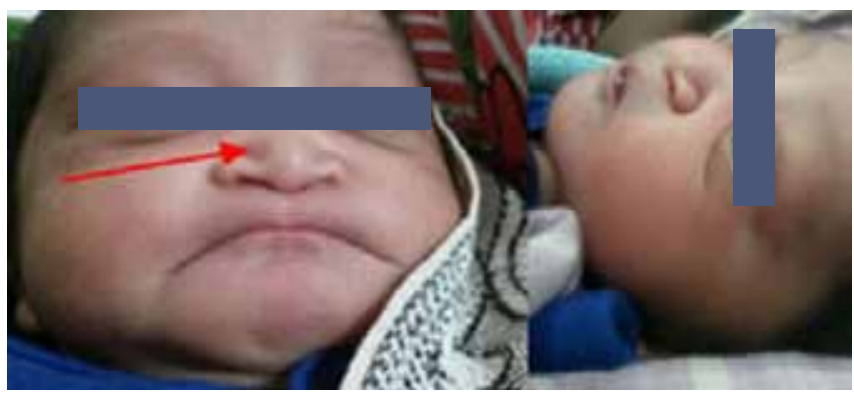

Fig.3: The characteristic Binder phenotype with absent nasal spine, semilunar alae nasi and convex upper lip. 
the need for karyotyping. Child was diagnosed with Binder syndrome secondary to warfarin intake in first trimester. Warfarin was restarted in dose of $6 \mathrm{mg}, 24$ hours after hematoma evacuation and continued with INR 2.2. Ecosprin 75 was also restarted. Patient was discharged on day 11 with the advice to start progesterone only pills at 4 weeks and close monitoring by pediatrician and need for orthodontics and plastic surgery in teen age. Patient is regular in follow up till date and both mother and child are doing well.

\section{Discussion}

Binder Syndrome is a rare congenital defect with varied etiologies, vitamin $\mathrm{K}$ deficiency due to warfarin intake being one of them. Individuals with Binder's syndrome have a characteristic appearance that is easily recognizable. The hypoplastic midface, flattened nose, convex upper lip with a broad philtrum, crescent or semi-lunar nostrils due to the short collumela, and a deep fold or fossa occuring between the upper lip and the nose, results in an acute nasolabial angle which can be seen in ultrasound [Fig.1].

Binder type maxillonasal dysplasia was first described in medical literature in 1882. $\mathrm{Dr}$ von Binder first identified the condition as a distinct clinical entity in 1962 and is hence named after him. It occurs in less than 1 in 10,000 live births $[1,2]$. Absent nasal bone seen in Binders is also seen in trisomy 21 which was first described in 1866, by Langdon Down [3]. The Binders syndrome closely resembles CDPR (chondrodysplasia punctata, rhizomelic type). Vitamin $\mathrm{K}$ deficiency is seen in both warfarin embryopathy and CDPR, in former because of competitive inhibition of coumarin derivative and in later because of mutation affecting enzyme aryl sulphatase. Warfarin interferes with the prenatal growth of the cartilaginous nasal septum by inhibiting the normal formation of a

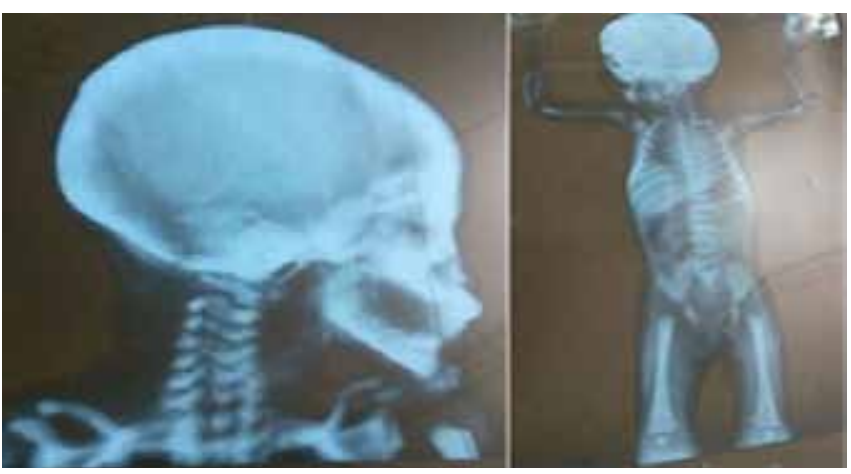

Fig.4: Neonate bone scan with normal stature, naso-maxillary hypoplasia and no epiphyseal stippling of long bones.

vitamin K-dependent protein Matrix Gla-protein (MGP) that prevents calcification of cartilage [3-5]. Warfarin intake leads to vitamin K deficiency between 6-9 weeks. Warfarin has a long halflife and remains in circulation for one month. Pre-conception counseling is thus important in patients with prosthetic valve taking warfarin. The thrombotic risk to mother is much less with warfarin but because of its associated embryopathy LMWH/ UFH should be initiated in early first trimester, at 6-9 weeks and warfarin resumed from 1236 weeks. Warfarin embryopathy is seen usually in dose requirement $>5 \mathrm{mg} /$ day. Daily dose of Vitamin $K$ should be replenished if patient is on parenteral therapy $[6,7]$.

Due to the rarity of the disease, there are no treatment trials for these patients. Treatments reported in the medical literature are part of single case reports or small case series of patients. Later, during adolescent period, the face can be surgically corrected. Nevertheless, some kids can have a natural correction. Intelligence is always normal. The prognosis of Binder phenotype, associated with other syndrome is dependent on the type of the associated syndrome. In some cases, the grafting of cartilage from the ribs has been successfully used to reconstruct the nose [8]. 


\section{Conclusion}

This case is discussed to highlight the rare disorder Binder syndrome which has a diverse etiology. There is need to shift from warfarin to UFH/LMWH at 6-9 weeks of conception in patients heart disease with mechanical valve prosthesis. Efforts should be made for pre-conception counseling by both treating cardiologist and obstetrician.

Acknowledgement: We would like to acknowledge Dr. Varun Duggal for performing obstetric ultrasound.

\section{References}

1. Cook K, Prefumo F, Presti, F, Homfray T, Campbell $\mathrm{S}$. The prenatal diagnosis of Binder syndrome before 24 weeks of gestation: case report. Ultrasound Obstet Gynecol. 2000; 1 6:578-581.

2. Binder $\mathrm{KH}$. Dysostosis maxillo-nasalis, einarhinencaphaler Missbildung skomplex Deutsch Zahnaertzl. 1962;17:438.

3. Howe AM, Webster WS, Lipson AH, Halliday JL,
Sheffield LJ. Binder's syndrome due to prenatal vitamin $\mathrm{K}$ deficiency: a theory of pathogenesis Aust Dent J. 1992;37(6):453-460.

4. Nedev PK. The Binder Syndrome: Review of The Literature And Case Report. The Internet Journal of Neurosurgery. 2008 Volume 5 Number 1.

5. NORD [National Organisation For rare Disoorders]. Binder type maxillonasal dysplasia Available at: https://rarediseases.org/rarediseases/binder-type-maxillonasal-dysplasia/ Accessed on December 16, 2015.

6. Toriello HV, Erick M, Alessandri JL. Maternal vitamin $K$ deficient embryopathy: association with hyperemesis gravidarum and Crohn disease. The American Journal of Medical Genetics. 2013;161(3):417-429.

7. Andrew S. Lane, Jennifer L. Stallworth, Kacey Y. Eichelberger, Kenneth F. Trofatter, Vitamin $K$ Deficiency Embryopathy from Hyperemesis Gravidarum. Case Reports in Obstetrics and Gynecology, vol. 2015, Article ID 324173, 3 pages, 2015.

8. Jain U, Thakur G, Kallury A. Binder's syndrome. BMJ Case Reports. $2011 ; 10: 1136$. 